\title{
Adaptive Fault Tolerant Control of UAV Attitude Control System
}

\author{
Hangzhou Zhao, Xu Yong, Yunqi Zhang and Yuhao Ding \\ School of Aerospace Engineering, Beijing Institute of Technology, China
}

\begin{abstract}
In this paper, we design a direct feedback adaptive fault-tolerant controller to cope with the uncertainty and non-modeling of the interference of the four-rotor UAV attitude control system. The controller is stuck in the UAV and the external. Under the condition of uncertainty, the attitude of the UAV can effectively track the state output of the reference system by introducing the feedback of the state and its error.
\end{abstract}

Keywords-UAV; state feedback; error adjustment; adaptive fault tolerant control

\section{INTRODUCTION}

With the development of UAV, improving the control quality of aircraft has become an important research content in the field of aircraft control and design field, ensuring that it can automatically identify faults, deal with the failure and interference has become a key link to improve the autonomy of the aircraft. Fault-tolerant control technology is a kind of advanced control method [1] which can be used to compensate the influence of failure and maintain the effect of system control when the control system has a certain range of fault interference. It has been widely used in the field of aerospace control [2-4].

Four rotor UAV flight state is changing at any time, the aerodynamic parameters are not accurate, the body dynamics is complex, the model library is imperfect, flight mechanism is nonlinear, variability and uncertainty, these unmodeled factors greatly increased UAV control difficulty. The attitude control system has the feature of over-drive, strong coupling and complex working conditions. The uncertainties of the flight process could also cause the sensor, the controller, the transmission link and the control system, once happened,actuators and other failures will do a fatal damage to the entire system. it is necessary to study the UAV external interference and fault compensation on the impact of the flight process.

In the present field of control, the nonlinear disturbance observer has been applied in many fields. Based on a class of nonlinear uncertain systems, it's main function is to observe uncertainties such as unmodeled impact of the system or unknown disturbances, so as to provide the design for the subsequent controller, the paper [5] design a robust tracking controller based on disturbance observer. According to the attitude control problem of rigid body satellites, in the paper [6], based on the output of disturbance observer, a fuzzy sliding mode interference compensation control method based on error quaternion is proposed. In the paper[7],an inversion control method based on sliding mode European technology is designed by using nonlinear observation jammers to approximate unmodeled dynamic and boundary unknown disturbances. In the paper [8] it propose an adaptive fault tolerant control system for the gain fault of the rotor, which can deal with the fault of the drive unit, but the lack of in-depth analysis of the fault detection, so it's no significance in practical application.

\section{PROBLEM DESCRIPTION}

\section{A. Mechanics Model of Four Rotor}

General, UAV use coordinate system is body coordinate system, ground coordinate system, and track coordinate system, Euler angle $((\psi, \theta, \phi)$,which represent the yaw angle, the pitch angle and the roll angle respectively, is used to describe the flight attitude, The generalized coordinate system of the UAV is $q=(x, y, z, \psi, \theta, \phi) \in R^{6}$, Where $(x, y, z)$ represents the distance from the fuselage to the origin of the inertial coordinate system, M1, M2, M3, M4 represent the four motors respectively, F1, F2, F3, F4 represent the lift force of four motors respectively. Firstly we need make a few assumptions [8]:

(1) UAV is a completely symmetrical rigid body structure.

(2) The output voltage of DC motor is linear with the output torque.

(3) The center of mass of UAV is completely coincident with the origin of the coordinate system.

(4) The ground coordinate system is the inertial coordinate system (ICS), the curvature of the earth can ignored, Acceleration of earth is a constant.

The scheme of a quad-rotor UAV is as follow:

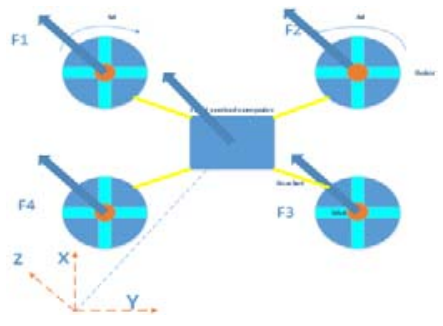

FIGURE I. THE SCHEME OF A QUAD ROTOR UAV

Let's assume it. $\xi=(\mathrm{x}, \mathrm{y}, \mathrm{z}) \in \mathrm{R}^{3}, \eta=(\psi, \theta, \phi) \in \mathrm{S}^{3}$, The linear kinetic energy, the angular momentum, and the gravitational potential energy of the four rotor UAV in the inertial space can be expressed as 


$$
\left\{\begin{array}{l}
T_{\text {trans }}=\frac{m}{2} \dot{\xi}^{T} \dot{\xi} \\
U=m g z \\
\text { Trot }=\frac{1}{2} \dot{\eta}^{T} J \dot{\eta}
\end{array}\right.
$$

Where m represent the quality of UAV, J represent the moment of inertia, g represent the gravity acceleration, then the Lagrangian equation of system could be as:

$$
L(q, \dot{q})=T_{\text {trans }}+T_{\text {rot }}-U=\frac{m}{2} \dot{\xi}^{T} \dot{\xi}+\frac{1}{2} \dot{\eta}^{T} J \dot{\eta}-m g z
$$

When $\frac{d}{d t} \frac{\partial L}{\partial \dot{q}}-\frac{\partial L}{\partial q}=F$, we can make a conclusion

$$
J \ddot{q}+\dot{J} q-\frac{\partial}{\partial q}\left(\frac{1}{2} \dot{q}^{T} J \dot{q}\right)=F
$$

Where $F=\left[F_{\xi}{ }^{T}, \tau^{T}\right]$ is the force of each channel, $\tau$ is the torque of each channel. Then there is next force equation: $F_{\xi}=R \tilde{F}$, where

\section{$R=\left[\begin{array}{ccc}\cos \theta \cos \phi & \sin \psi \sin \theta & -\sin \theta \\ \cos \psi / \sin \theta \sin \phi-\sin \psi \cos \phi & \sin \psi \sin \theta \sin \phi+\cos \psi \cos \phi & \cos \theta \sin \psi \\ \cos / / \sin \theta \cos \phi-\sin \psi \sin \phi & \sin \psi \sin \theta \cos \phi-\cos \psi \cos \phi & \cos \theta \sin \gamma\end{array}\right]$}

Is represent the coordinate transformation function matrix,

$$
\widetilde{F}=\left[\begin{array}{c}
0 \\
0 \\
F_{1}+F_{2}+F_{3}+F_{4}
\end{array}\right] \text { is represent the lift force of }
$$

rotor. According to Newton Law

$$
\begin{aligned}
& \tau=\left[\begin{array}{c}
\tau_{\psi} \\
\tau_{\theta} \\
\tau_{\phi}
\end{array}\right]=\left[\begin{array}{c}
K_{t c}\left(V_{3}+V_{4}\right)+K_{t n}\left(V_{1}+V_{2}\right) \\
K_{f} l\left(V_{1}-V_{2}\right) \\
K_{f} l\left(V_{3}-V_{4}\right)
\end{array}\right] \\
& J \ddot{\eta}+\dot{J} \dot{\eta}-\frac{\partial}{\partial \eta}\left(\frac{1}{2} \dot{\eta}^{T} J \dot{\eta}\right)=\tau \text {, then Coriolis vector }
\end{aligned}
$$

can be represented as follow:

$$
\begin{aligned}
& \bar{V}(\eta, \dot{\eta})=\dot{J} \dot{\eta}-\frac{1}{2} \frac{\partial}{\partial \eta}\left(\dot{\eta}^{T} J \dot{\eta}\right) \\
& =\left(\dot{J}-\frac{1}{2} \frac{\partial}{\partial \eta}\left(\dot{\eta}^{T} J\right)\right) \dot{\eta}=C(\eta, \dot{\eta}) \dot{\eta}
\end{aligned}
$$

In order to simplify the operation, we can make a command: $\tau=C(\eta, \ddot{\eta}) \dot{\eta}+J \tilde{\tau}$, then $\ddot{\eta}=\tilde{\tau}$.

In this paper, we doing lots of research on each system of UAV respectively, on the base of follow assumption, the attitude change angle is not more than 5 degrees, $\dot{J}=0, \theta=\phi=\psi \approx 0$, so the axis equation of $\mathrm{UAV}$ is just as follow:

$$
J \ddot{\eta}=\left[\begin{array}{c}
J_{y} \ddot{\psi} \\
J_{p} \ddot{\theta} \\
J_{r} \ddot{\phi}
\end{array}\right]=\left[\begin{array}{c}
K_{t c}\left(V_{3}+V_{4}\right)+K_{t n}\left(V_{1}+V_{2}\right) \\
K_{f} l\left(V_{1}-V_{2}\right) \\
K_{f} l\left(V_{3}-V_{4}\right)
\end{array}\right]
$$

So the control input vector is $u(t)=\left(V_{1}, V_{2}, V_{3}, V_{4}\right)^{T}$, the state variable of the system is $x=(\psi, \theta, \phi, \dot{\psi}, \dot{\theta}, \dot{\phi})^{T}$, the output vector is $y=(\psi, \theta, \phi)^{T}$, so the System state space

Expression can be as:

$$
\left\{\begin{array}{l}
\dot{x}(t)=A x(t)+B u(t) \\
y(t)=C(t)+D u(t)
\end{array}\right.
$$

In which $A$ is state matrix, $B$ is input matrix, $C$ is output matrix, $\mathrm{D}$ is incidence. Where

$$
\begin{aligned}
A & =\left[\begin{array}{cccccc}
0 & 0 & 0 & 1 & 0 & 0 \\
0 & 0 & 0 & 0 & 1 & 0 \\
0 & 0 & 0 & 0 & 0 & 1 \\
0 & 0 & 0 & 0 & 0 & 0 \\
0 & 0 & 0 & 0 & 0 & 0 \\
0 & 0 & 0 & 0 & 0 & 0
\end{array}\right], \quad C=\left[\begin{array}{llllll}
1 & 0 & 0 & 0 & 0 & 0 \\
0 & 1 & 0 & 0 & 0 & 0 \\
0 & 0 & 1 & 0 & 0 & 0
\end{array}\right] \\
B & =\left[\begin{array}{cccc}
0 & 0 & 0 & 0 \\
0 & 0 & 0 & 0 \\
\frac{K_{t c}}{J_{y}} & \frac{K_{t c}}{J_{y}} & \frac{K_{t n}}{J_{y}} & \frac{K_{t n}}{J_{y}} \\
\frac{I K_{f}}{J_{p}} & -\frac{I K_{f}}{J_{p}} & 0 & 0 \\
0 & 0 & \frac{I K_{f}}{J_{r}} & -\frac{I K_{f}}{J_{r}}
\end{array}\right], \quad D=\left[\begin{array}{lll}
0 & 0 & 0 \\
0 & 0 & 0 \\
0 & 0 & 0
\end{array}\right]
\end{aligned}
$$

\section{B. Control Problem Description}

Face with the case of external disturbance and actuator failure, the model of the attitude control system of four rotor UAV with three degrees of freedom can be described:

$$
\dot{x}=(A+\Delta A) x(t)+B u(t)+B_{\omega} \omega(t)
$$


Where $\Delta A$ is represent the parameter uncertainty of the UAV system, $B_{\omega} \in R^{n \times n_{\omega}}$ is represent the interference signal of Parameter matrix, $\omega(t)$ is represent the external interference signal. These parameters satisfy the following formula:

$$
\Delta A=\mathrm{BN}(t), B_{\omega}=B F .
$$

Usually $\mathrm{N}(t)$ is unknown but is limited, $\mathrm{F}$ is not only known but also is limited $\left.\|N(t)\|<=l^{*}, \| F\right) \|<=\lambda_{f}$.

In this paper. Stuck fault, partial fault and unknown fault are taken into account. Now let's assume that $u_{i j}^{F}$ represent the fault input signal when the actuator i occur fault type $j$, $\mathrm{u}_{\mathrm{i}}(t)$ is defined as the input signal when when the actuator $\mathrm{i}$ is on good condition, $\mathrm{u}_{\mathrm{si}}(t)$ is defined as the input signal when when the actuator $\mathrm{i}$ is stuck dead. So the input signal of fault can be replaced by

$$
u_{i j}^{F}=\rho_{i}^{j} u_{i}(t)+\sigma_{i}^{j} u_{s i}(t), i=1,2, \ldots, m, j=1,2, \ldots, L
$$

Where $\mathrm{u}_{j}(t)=\overline{\mathrm{u}_{j}}, \quad t>\mathrm{t}_{j}, j \in\{1,2, \ldots, m\}$. in which $\rho_{i}^{j} \in[0,1]$ indicates the extent of the failure in the actuator, $\sigma_{i}^{j} \in[0,1]$ indicates the extent to which the agency is stuck, and $\mathrm{L}$ indicates that the total number of failures that may occur in the actuator, when actuator i get stuck, the fault input signal can be regarded as:

$$
u_{i}^{F}=\rho_{\mathrm{i}} u_{i}(t)+\sigma_{\mathrm{i}} u_{s i}(t), \rho_{\mathrm{i}} \in\left\{\rho_{i}^{1}, \rho_{i}^{2}, \ldots, \rho_{i}^{L}\right\}, \sigma_{\mathrm{i}} \in\left\{\sigma_{i}^{1}, \sigma_{i}^{2}, \ldots, \sigma_{i}^{L}\right\}
$$

So the total input fault is

$$
u^{F}=\rho u_{i}(t)+\sigma u_{s}(t)
$$

in which

$$
\left\{\begin{array}{l}
\rho=\operatorname{diag}\left(\rho_{1}, \rho_{2}, \ldots, \rho_{m}\right) \\
\sigma=\operatorname{diag}\left(\sigma_{1}, \sigma_{2}, \ldots, \sigma_{m}\right) \\
u_{s}(t)=\left[u_{s 1}(t), u_{s 2}(t), \ldots, u_{s n}(t)\right]^{\mathrm{T}}
\end{array},\right.
$$

in the last ,the system model can be described as

$$
\dot{x}=(A+\Delta A) x(t)+B\left(\rho u_{i}(t)+\sigma u_{s}(t)\right)+B_{\omega} \omega(t)
$$

The target of this paper is design an adaptive fault tolerant controller(FTC) based on state feedback and error adjustment, so that the UAV can keep the state signal $x(t)$ under certain conditions, where the system model is not established, the actuator failure and interference are uncertain , the state signal could still track the state of the reference system $x_{m}(t)$.

The state of the reference system is as follow:

$$
\dot{x}_{m}(t)=A_{m} x_{m}(t)+B_{m} r(t)
$$

$A_{m}, B_{m}$ represent the state matrix and control matrix of the reference system, respectively, the reference system is on stably. All the characteristic roots of $A_{m}$ are located in the left axis of the complex plane, $r(t) \in \mathrm{R}^{l}$ is uniformly continuous and limited, which is input signal of the reference system.

We can assume: in any failure, mode $\left\{A, B_{\rho}\right\}$ is completely controllable, namely, there are matrix, $A+B \rho K_{1}^{* T}=A_{m}$, and, $B \rho K_{2}^{* T}=B_{m}$. The stuck fault of actuator and the external disturbance is continuous segmented and limited. There is a matrix $K_{3}^{*} \in \mathrm{R}^{\mathrm{m} \times \mathrm{l}}$ and a constant

$$
\begin{aligned}
& B \rho K_{3}^{*}+B \rho u_{s}=0 \\
& \|\omega(t)\|<=\bar{\omega}
\end{aligned}
$$

They are satisfy

$$
\|\mathrm{F} \omega(t)\|<=\|F \mid\| \omega(t)\|<=\| F \| \bar{\omega}<=u l_{1}^{*}
$$

If any actuator get fault, the remaining controller can still guarantee the realization of the control target. Then there are

$$
\left\|e^{T} P B \rho B^{T} P e\right\|>=\mu\left\|e P^{T} B\right\|^{2}
$$

\section{Adaptive Fault Tolerant Controller Design}

The structure of adaptive fault-tolerant control system is just as follow:

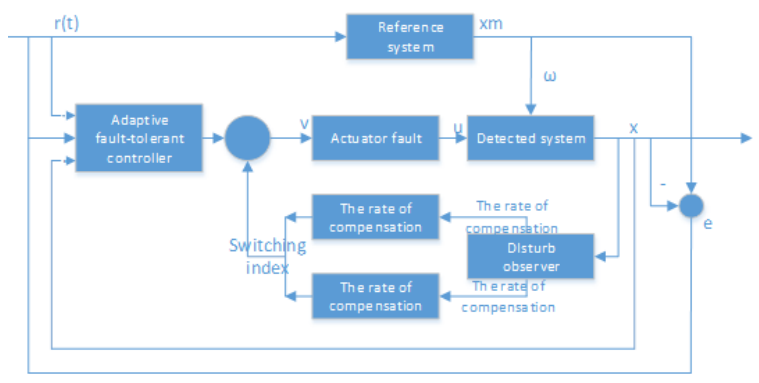

FIGURE II. THE STRUCTURE OF ADAPTIVE FAULT-TOLERANT CONTROL SYSTE

Firstly, we can state the tracking error signal:

$$
e(t)=x(t)-x_{m}(t)
$$


In this paper, the target controller can be defined as:

$$
u(t)=K_{1}^{T} x(t)+K_{2}^{T} r(t)+k_{3}^{*}+\left(k_{4}+k_{5}\right) e(t)
$$

Where $k_{1}, k_{2}, k_{3}$ were assumed to be the estimation of $k_{1}^{*}, k_{2}^{*}, k_{3}^{*}, k_{4}, k_{5}$ were assumed to be the error feedback matrix. There were used to compensate the influence of the system uncertainties and external disturbances on the system.

When the designed controller replaced into the controlled object.

$$
\begin{aligned}
& \dot{x}=\left[A+B \rho K_{1}^{T}\right] x(t)+B \rho K_{2}^{T} r(t)+B \rho K_{3} \\
& +\Delta A x(t)+B \rho\left(K_{4}+K_{5}\right) e(t)+B \rho u_{s}(t)+B_{\omega} \omega(t)
\end{aligned}
$$

The error variable for the system is

$$
\left\{\begin{array}{l}
\overline{\mathrm{k}}_{1}(t)=k_{1}(t)-k_{1}^{*} \\
\overline{\mathrm{k}}_{2}(t)=k_{2}(t)-k_{2}^{*} \\
\overline{\mathrm{k}}_{3}(t)=k_{3}(t)-k_{3}^{*}
\end{array}\right.
$$

Then the state change of controller is

$$
\begin{aligned}
& \dot{x}=A_{m} x(t)+B_{m} r(t)+B \rho\left(\tilde{K}_{1}^{T} x+\tilde{K}_{2}^{T} x+\tilde{K}_{3}\right) \\
& +\Delta A x(t)+B \rho\left(k_{4}+k_{5}\right) e(t)+B_{\omega} \omega(t)
\end{aligned}
$$

Once replaced the state change of controller with the derivative of tracking error, we can get this:

$$
\begin{aligned}
& \dot{e}(t)=\dot{x}(t)-\dot{x}_{m}(t) \\
& =A_{m}(t)+B_{m}(t)+B \rho\left(\tilde{K}_{1}^{T} x+\tilde{K}_{2}^{T} \gamma+\tilde{K}_{3}\right) \\
& +\Delta A x(t)+B \rho\left(K_{4}+K_{5}\right) e(t)+B_{\omega}(t)
\end{aligned}
$$

According to the regulation of adaption: $k_{1 j}, k_{2 j}, k_{3 j}, j=1,2, \ldots, m$ should be designed as:

$$
\left\{\begin{array}{l}
\dot{k}_{1 j}=\dot{\widetilde{k}}_{1 j}=-\Gamma_{1 j} x(t) e^{T}(t) P b_{j} \\
\dot{k}_{2 j}=\dot{\widetilde{k}}_{2 j}=-\Gamma_{2 j} x(t) e^{T}(t) P b_{j} \\
\dot{k}_{3 j}=\dot{\widetilde{k}}_{3 j}=-\gamma_{3 j} e^{T}(t) P b_{j}
\end{array}\right.
$$

In

$\Gamma_{1 j} x(t)=\Gamma_{1 j}^{\mathrm{T}} x(t)>0, \quad \Gamma_{, 2 j} x(t)=\Gamma_{2 j}^{\mathrm{T}} x(t)>0$

which arbitrary constant matrix, $\gamma_{3 j}>0$ is arbitrary constant, $P=P^{T} \in R^{n \times n}$ is arbitrary constant positive symmetric matrix, For any arbitrary constant positive symmetric matrix $Q$, there is $A_{m} P+P A_{m}+\frac{1}{\eta}(1+\varepsilon)=-Q, \varepsilon>0$.

We can introduce a system parameter $l_{2}^{*}=l^{2} / u$. According to the the paper [10],we can make a conclusion; the parameter can should set to be

$$
\left\{\begin{array}{l}
k_{4}=\frac{l_{1}^{2} B^{T} P}{\left\|e^{T} P B\right\| l_{1}+\delta(t)} \\
k_{5}=-\frac{1}{2} \eta l 2 B^{T} P
\end{array}\right.
$$

Where $\delta(t) \in \mathrm{R}^{+}$is is an arbitrary uniformly continuous and limited function, and $\lim _{t \rightarrow \infty} \int_{t 0}^{\mathrm{t}} \delta(t) d \tau<=\bar{\delta}<\propto, l_{1}, l_{2}$ is the estimated value of $l_{1}^{*}, l_{2}^{*}$ respectively, The adaptive rate can be expressed as

$$
\left\{\begin{array}{l}
\frac{d l_{1}}{d t}=-\gamma_{4} \delta(t) l_{1}+2 \gamma_{4}\|e T P B\| \\
\frac{d l_{2}}{d t}=-\gamma_{5} \delta(t) l_{2}+2 \gamma_{5}\|e T P B\|^{2}
\end{array}\right.
$$

The error were defined as

$$
\left\{\begin{array}{l}
\tilde{l}_{1}=l_{1}-l_{1}^{*} \\
l_{2}=l_{2}-l_{2}^{*}
\end{array}\right.
$$

There a law: for closed loop system (9) , if it's control structure is expressed as (11), the control rate is expressed as (22), the parameter adaptive rate is expressed as(21) and (23). Then we can make conclude that the state of the controlled system is limited that can track the output state of the reference system.

\section{THEORETICAL DEMONSTRATION}

Proof: we can construct a L yapunov function [12]

$$
\begin{aligned}
& \dot{V}_{p}=e^{T} P e+\sum_{j=1}^{m}\left(p_{j} \tilde{k}_{1}^{T} \Gamma_{1 j}^{-1} k_{1 j}+p_{j} \tilde{k}_{2}^{T} \Gamma_{2 j}^{-1} k_{2 j}+\rho_{j} \tilde{k}_{3}^{2} \Gamma_{3 j}^{-i}\right) \\
& +\frac{1}{2} u\left(\gamma_{4}^{-1} \widetilde{l}_{1}^{2}+\gamma_{5}^{-1} \tilde{l}_{2}^{2}\right)
\end{aligned}
$$

We can take the derivative of the above formula

$$
\begin{aligned}
& \dot{V}_{p}=2 e^{T} P A_{m} e+2 e^{T} P \Delta A x(t)+2 e^{T} P B \rho\left(k_{4}+k_{5}\right) e(t) \\
& +2 e^{T} P B_{\omega} \omega(t)+u\left(\gamma_{4}^{-1} \tilde{\tilde{l}}_{1}+\gamma_{5}^{-1} \tilde{\tilde{l}_{2}} \dot{\tilde{l}}_{2}\right)
\end{aligned}
$$


Because

$\|x\|^{2}=\left(e+x_{m}\right)^{T}\left(e+x_{m}\right)=\|e\|^{2}+\left\|x_{m}\right\|^{2}+2 e^{T} x_{m}$

$<=(1+\varepsilon)\|e\|^{2}+\left(1+\varepsilon^{-1}\right)\left\|x_{m}\right\|^{2}<=(1+\varepsilon)\|e\|^{2}+\left(1+\varepsilon^{-1}\right) \lambda_{m}$

is on the ground. So

$$
\begin{aligned}
& 2 e^{T} P \Delta A x=2 e^{T} P B N(t) x<=2 l^{*}\left\|e^{T} P B\right\| x \| \\
& <=l^{*}\left[\frac{1}{\eta l^{*}}\|x\|^{2}+\eta l^{*}\left\|e^{T} P B\right\|^{2}\right] \\
& <=\frac{1}{\eta}\left[(1+\varepsilon)\|e\|^{2}+\left(1+\varepsilon^{-1}\right) \lambda_{m}\right]+\eta l^{*}\left\|e^{T} P B\right\|^{2} \\
& =\frac{1}{\eta}\left[(1+\varepsilon)\|e\|^{2}+\left(1+\varepsilon^{-1}\right) \lambda_{m}\right]+u \eta l^{*}\left\|e^{T} P B\right\|^{2}
\end{aligned}
$$

Where $\eta>0$. according to the formula (14),

$$
2 e^{T} P B \omega(t)=2 e^{T} P B \operatorname{ra}(t)<=2\left\|e^{T} P B\right\| F|| \bar{\omega}<=2 \mid e^{T} P \not \| u_{1}^{*}
$$

We can take formula (27) and (28) into (25)

$$
\begin{aligned}
& \dot{V}_{p}<=e^{T}\left[A_{m}^{T} P+P A_{m}+\frac{1}{\eta}(1+\varepsilon)\right] e+u \eta l_{2}^{*} \|\left. e^{T} P B\right|^{2} \\
& +2 u l_{1}^{*} \| e^{T} P B+\frac{1}{\eta}\left(1+\varepsilon^{-1}\right) \lambda_{m}+2 e^{T} P B p\left(k_{4}+k_{5}\right) e \\
& +u\left(\gamma_{4}^{-1} \tilde{\tilde{l}}_{1} \dot{\vec{l}}_{1}+\gamma_{5}^{-1} \dot{\tilde{\vec{l}}}_{2}\right)
\end{aligned}
$$

Then we take (22) into the control rate (15).

$$
\begin{aligned}
& \dot{V}_{p}<=e^{T}\left[A_{m}^{T} P+P A_{m}+\frac{1}{\eta}(1+\varepsilon)\right] e+\frac{1}{\eta}\left(1+\varepsilon^{-1}\right) \lambda_{m} \\
& +u \eta l_{2}^{*}\left\|e^{T} P B\right\|^{2}+2 u l_{1}^{*}\left\|e^{T} P B\right\|-\frac{2 u l_{1}\left\|e^{T} P B\right\|^{2}}{\left\|e^{T} P B\right\| l_{1}+\delta(t)} \\
& -u \eta l_{2}\left\|e^{T} P B\right\|^{2}+u\left(\gamma_{4}^{-1} \tilde{l}_{1} \dot{\vec{l}}_{1}+\gamma_{5}^{-1} \tilde{l}_{2} \dot{\tilde{l}}_{2}\right)
\end{aligned}
$$

According to (23), the formula (22) can be replaced with

$$
\left\{\begin{array}{l}
\frac{d \tilde{l}_{1}}{d t}=-\gamma_{4} \delta(t) \tilde{l}_{1}+2 \gamma_{4} \eta\left\|e^{T} P B\right\|-\gamma_{4} \delta(t) l_{1}^{*} \\
\frac{d \tilde{l}_{2}}{d t}=-\gamma_{5} \delta(t) \tilde{l}_{2}+2 \gamma_{5} \eta\left\|e^{T} P B\right\|-\gamma_{5} \delta(t) l_{2}^{*}
\end{array}\right.
$$

\footnotetext{
We take (6-42) into (6-41), the get
}

$$
\begin{aligned}
& \dot{V}_{p}<=e^{T}\left[A_{m}^{T} P+P A_{m}+\frac{1}{\eta}(1+\varepsilon)\right] e+\frac{1}{\eta}\left[\left(1+\varepsilon^{-1}\right) \lambda_{m}\right. \\
& +u \eta l_{2}^{*}\left\|e^{T} P B\right\|^{2}+2 u l_{1}^{*}\left\|e^{T} P B\right\|+\frac{2 u\left\|e^{T} P B\right\|^{2} l_{1} \delta(t)}{\left\|e^{T} P B\right\| l_{1}+\delta(t)} \\
& -u \delta(t)\left(\tilde{l}_{1}^{2}+\tilde{l}_{1} l_{1}^{*}+\tilde{l}_{2}^{2}+\tilde{l}_{2} l_{2}^{*}\right)
\end{aligned}
$$

Because of $A_{m} P+P A_{m}+\frac{1}{\eta}(1+\varepsilon)=-Q, \varepsilon>0$, then we can get

$$
\begin{aligned}
& \dot{V}_{p}<=e^{T}\left[A_{m}^{T} P+P A_{m}+\frac{1}{\eta}(1+\varepsilon)\right] e+\frac{1}{\eta}\left(1+\varepsilon^{-1}\right) \lambda_{m} \\
& +u \eta l_{2}^{*}\left\|e^{T} P B\right\|^{2}+2 u l_{1}^{*}\left\|e^{T} P B\right\|+\frac{2 u\left\|e^{T} P B\right\|^{2} l_{1} \delta(t)}{\left\|e^{T} P B\right\| l_{1}+\delta(t)} \\
& -u \delta(t)\left(\tilde{l}_{1}^{2}+\tilde{l}_{1}^{*} l_{1}^{*}+\tilde{l}_{2}^{2}+\tilde{l}_{2} l_{2}^{*}\right)<=-e^{T} Q e+\frac{1}{\eta}\left(1+\varepsilon^{-1}\right) \lambda_{m} \\
& +\frac{2 u\left\|e^{T} P B\right\|^{2} l_{1} \delta(t)}{\left\|e^{T} P B\right\| l_{1}+\delta(t)}+u \delta(t)\left(\tilde{l}_{1}^{2}+\tilde{l}_{1} l_{1}^{*}+\tilde{l}_{2}^{2}+\tilde{l}_{2} l_{2}^{*}\right)
\end{aligned}
$$

We can simplify the formula as follow according to the formula $0<=\frac{a b}{a+b}<=a, \forall a, b>0$,

$$
\begin{aligned}
& \dot{V}_{p}<=-e^{T} Q e+\frac{1}{\eta}\left(1+\varepsilon^{-1}\right) \lambda_{m}+2 u \delta(t)+u \delta(t)\left(\frac{l_{1}^{* 2}}{4}+\frac{l_{2}^{* 2}}{4}\right) \\
& <=-\lambda \min \|\left.\right|^{2}+\frac{1}{\eta}\left(1+\varepsilon^{-1}\right) \lambda_{m}+\delta(t) u\left(2+\frac{l_{1}^{* 2}}{4}+\frac{l_{2}^{* 2}}{4}\right) \\
& <=-\lambda \min \|\left.\right|^{2}+\frac{1}{\eta}\left(1+\varepsilon^{-1}\right) \lambda_{m}+\delta(t) \kappa
\end{aligned}
$$

Where

$$
\kappa=\mathrm{u}\left(2+\frac{l_{1}^{* 2}}{4}+\frac{l_{2}^{* 2}}{4}\right)
$$

IV. SIMULATION AND ANALYSIS

The parameters of the reference system are as follows:

$$
A_{n}=\left[\begin{array}{cccccc}
0 & 0 & 0 & 1 & 0 & 0 \\
0 & 0 & 0 & 0 & 1 & 0 \\
0 & 0 & 0 & 0 & 0 & 1 \\
-605 & 0 & 0 & -3.05 & 0 & 0 \\
0 & -6004 & 0 & 0 & -2005 & 0 \\
0 & 0 & -6010 & 0 & 0 & -2005
\end{array}\right], B_{m}=\left[\begin{array}{cc}
0 & 0 \\
0 & 0 \\
0 & 0 \\
4 & -1 \\
4 & 4 \\
0 & 0
\end{array}\right]
$$




$$
\begin{aligned}
& N(t)=\left[\begin{array}{cccccc}
0 & 0 & 0 & -0.03 & -0.35 & 0.13 \\
0 & 0 & 0 & 0.03 & -0.35 & 0.13 \\
0 & 0 & 0 & 0.05 & 0.35 & -0.13 \\
0 & 0 & 0 & 0.05 & 0.35 & -0.13
\end{array}\right] \quad F=I_{4}, \\
& u_{s}(t)=[0.1,0.2,0.2,0.1]^{T}, \mathrm{Q}=10 * I_{6}, \Gamma_{1 i}=10 * I_{6}, \\
& \Gamma_{2 i}=10 * I_{2}, \gamma_{3 i}=20, \gamma_{4}=\gamma_{5}=0.05, \eta=100 . \\
& \text { the } \quad \text { Simulation: } \\
& x(o)=x_{m}(0)=[0,0,0,0,0,0]^{T}, \quad k_{1 i}(0)=[0,0,0,0,0,0]^{T}, \\
& x(o)=x_{m}(0)=[0,0,0,0,0,0]^{T} \quad, \quad k_{2 i}(0)=[5,-5]^{T}, \\
& k_{3}(0)=[1,1,-1,-1]^{T}, l_{1}(0)=l_{2}(0)=0 .
\end{aligned}
$$

According to the above parameters and initial conditions, the tracking error of the attitude angle of the four rotor UAV can be obtained as follows[8]:
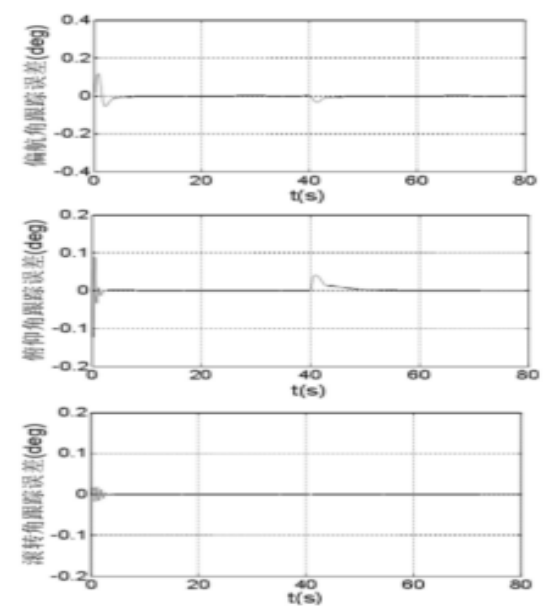

FIGURE III. ATTITUDE ANGLE ERROR BEFORE INTERFERENCE
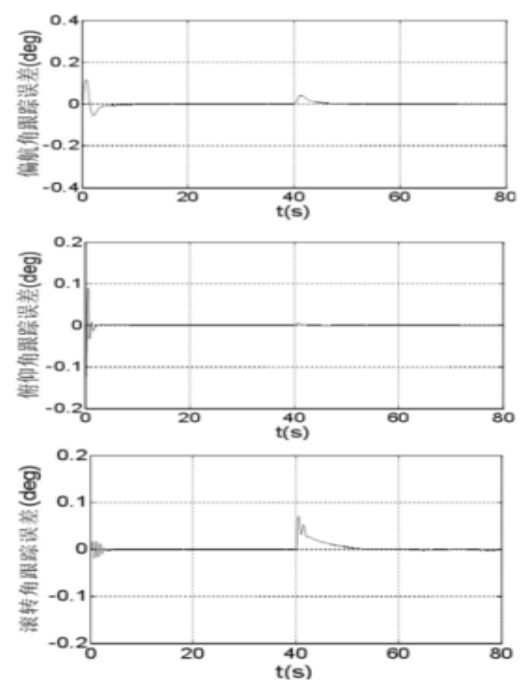

FIGURE IV. ATTITUDE ANGLE ERROR AFTER INTERFERENCE

We assume that the system fails at $t=40$ s.In the fig1, $\rho=\operatorname{diag}(0,1,1,1), \sigma=\operatorname{diag}(1,0,0,0)$, actuator $u_{1}$ get stuck fault,
$\mathrm{UAV}$ is under the control of $\boldsymbol{u}_{3}, \boldsymbol{u}_{4}$, Only the yaw angle and the pitch angle tracking error appear obvious transient response, but the roll angle is not get affected obviously. In the fig2, $\rho=\operatorname{diag}(1,1,0,1), \sigma=\operatorname{diag}(0,0,0,0)$, actuator $u_{3}$ get stuck fault, when it goes to $40 \mathrm{~s}, \mathrm{UAV}$ is under the control of $u_{1}, u_{2}$, Only the yaw angle and the roll angle tracking error appear obvious transient response, but the pitch angle is not get affected obviously.

Under the action of Adaptive fault tolerant controller in this paper, the tracking error decreases rapidly, and stay in a small community, or even close to zero. simulation results show that the algorithm is effective and has good performance. compare with the controller in the paper [11], we introduce the feedback of reference system state, and then get a fault tolerant control system with excellent control performance.

\section{V.SUMMARY}

Faced with uncertainty of system parameters of linear continuous system, the paper[11] design a kind of robust adaptive algorithm, which can be used to compensate the actuator faults and external disturbances. on the base of that paper, we do some research on the problem of fault tolerant control of four rotor helicopter attitude control system with uncertainty of system parameters, and then design a kind of adaptive fault tolerant controller, which can use to track the state of the reference system, we also use the Lyapunov stability theory to analyze the performance of the system. Finally, through the simulation results, it is verify the effectiveness and feasibility of the proposed algorithm.

\section{REFERENCE}

[1] Xiaodong Zhang,T.Parisini,M.M. Polycarpou.Adaptive fault tolerant control of nonlinear uncertain systems: An information based diagnostic approach[J].IEEE TRANSACTION ON AUTOMTIC CONTROL, VOL.49, NO.8, AUGUST 2004

[2] Jovan D. Boskovic and Raman K. Mehra. Robust fault tolerant control design for aircraft under state dependent disturbances[J].AIAA Guidance, Navigation, and Control Conference and Exhibit11-14 August 2003, Austin, Texas

[3] BOSKOVIC J D, MEHRARK. A Decentralized fault tolerant control system for accommodation of failures in higher order flight control actuators[J]. IEEE Transactions on Control System Technology, 201018 (5) : 1103-1115

[4] Chun Liang Lin, Senior Member, IEEE, and Chun-Te Liu. Failure Detection and Adaptive Compensation for Fault Tolerable Flight Control Systems: IEEE TRANSACTIONS ON INDUSTRIAL INFORMATICS, VOL. 3, NO. 4, NOVEMBER 2007

[5] Diao C, Xian B, Yin Q. A nonlinear adaptive control approach for quad rotor UAVS[C]. Control Conference(ASCC), 2011:223-228.

[6] Chau Mu, Chagrin Sun, Changsha Qian. Four-element Fuzzy Membrane Control of Satellite Attitude Error Based on Disturbance Observer [J]. Nanjing: Southeast University, 2012, 42(5):886-891.

[7] Song hay, Grog Zhao, Jun Feng Gay. Inverse Sliding Mode Control of Hypersonic Vehicle Based on Nonlinear Interference Observer [J]. Systems Engineering and Electronics, 2013,34(6) : 1231-1234

[8] Rabab Ran Kho Res nick. Generalized fault recovery of an under-actuated quad rotor aerial vehicle Proceedings of the American Control conference Montreal Canada 2012: 2515-2520.

[9] Fief Lu. Adaptive fault - tolerant algorithm for four - rotor helicopter attitude control system [D]. Nanjing: Nanjing University of Aeronautics and Astronautics, 2014 
[10] Tao Shu. Hal. Chen, X. D. Tang, Adaptive Control of Systems with Actuator Failures [M]. Sprinter, March, 2004.

[11] Li X J, Yang G H. Robust adaptive fault-tolerant control for uncertain linear systems with actuator failures [J]. Control Theory and Applications, IET,2012,6(10): 1544-1551

[12] Nib He, Changchun Chang. I. Adaptive observer design of nonlinear system based on Lyapunov method [J]. Nanjing: Nanjing University of Aeronautics and Astronautics, 2006,38(3):267-270 